PROCEEDINGS OF THE

AMERICAN MATHEMATICAL SOCIETY

Volume 134, Number 8, Pages 2411-2418

S 0002-9939(06)08554-6

Article electronically published on March 21, 2006

\title{
RADIX REPRESENTATIONS, SELF-AFFINE TILES, AND MULTIVARIABLE WAVELETS
}

\author{
EVA CURRY
}

(Communicated by Jonathan M. Borwein)

\begin{abstract}
We investigate the connection between radix representations for $\mathbb{Z}^{n}$ and self-affine tilings of $\mathbb{R}^{n}$. We apply our results to show that Haar-like multivariable wavelets exist for all dilation matrices that are sufficiently large.
\end{abstract}

\section{INTRODUCTION}

We investigate the connections between radix representations for $\mathbb{Z}^{n}$, self-affine tilings of $\mathbb{R}^{n}$, and Haar-like scaling functions for multiresolution analyses and associated wavelet sets.

In a separate paper, we investigate the idea, also introduced by Jeong [5], of radix representations for vectors in $\mathbb{Z}^{n}$ or general point lattices $\Gamma=M\left(\mathbb{Z}^{n}\right)(M$ a nondegenerate $n \times n$ matrix) [1]. We wish to consider expanding matrices which preserve $\Gamma$. Without loss of generality, we may assume $\Gamma=\mathbb{Z}^{n}$. A matrix which preserves $\mathbb{Z}^{n}$ must have integer entries.

Definition 1. A dilation matrix for $\mathbb{Z}^{n}$ is an $n \times n$ matrix $A$ with integer entries, all of whose eigenvalues $\lambda$ satisfy $|\lambda|>1$.

Note that for a dilation matrix $A, q:=|\operatorname{det} A|$ is an integer, with $q>1$. Then $\mathbb{Z}^{n} / A\left(\mathbb{Z}^{n}\right)$ has a nontrivial cokernel. Let $D$ be a complete set of coset representatives of $\mathbb{Z}^{n} / A\left(\mathbb{Z}^{n}\right)$. We call the elements of $D$ digits.

We may associate a sequence of digits with each $x \in \mathbb{Z}^{n}$ by the Euclidean algorithm, as follows. Each $x \in \mathbb{Z}^{n}$ is in a unique coset of $\mathbb{Z}^{n} / A\left(\mathbb{Z}^{n}\right)$, thus there exist unique $x_{1} \in \mathbb{Z}^{n}$ and $r_{0} \in D$ such that

$$
x=A x_{1}+r_{0} .
$$

Similarly, for each $x_{j}, j \geq 1$, there exist unique $x_{j+1} \in \mathbb{Z}^{n}$ and $r_{j} \in D$ such that

$$
x_{j}=A x_{j+1}+r_{j} \text {. }
$$

Formally, we write

$$
x \sim \sum_{j=0}^{\infty} A^{j} r_{j} .
$$

Received by the editors March 9, 2005.

2000 Mathematics Subject Classification. Primary 52C22, 42C40; Secondary 11A63.

Key words and phrases. Self-affine tiling, radix representation, multivariable wavelet, Haar-like wavelet, dilation matrix.

(C)2006 American Mathematical Society Reverts to public domain 28 years from publication 
If there exists a nonnegative integer $N$ such that $r_{j}=\mathbf{0}$ for all $j>N$, then the Euclidean algorithm terminates and we say that $x$ has a radix representation with radix $A$ and digit set $D$.

Definition 2. Let $A$ be a dilation matrix. We say that the matrix $A$ yields a radix representation with digit set $D$ if for every $x \in \mathbb{Z}^{n}$ there exists a nonnegative integer $N=N(x)$ and a sequence of digits $d_{0}, d_{1}, \ldots, d_{N}$ in $D$ such that

$$
x=\sum_{j=0}^{N} A^{j} d_{j} \text {. }
$$

That is, a dilation matrix $A$ yields a radix representation with digit set $D$ if every $x \in \mathbb{Z}^{n}$ has a radix representation with radix $A$ and digit set $D$.

Let $A$ be a dilation matrix, and define

$$
\mu=\min \{\sigma: \sigma \text { a singular value of } A\} \text {. }
$$

Let $F$ be a fundamental domain for $\mathbb{Z}^{n}$, centered at the origin,

$$
F=\left[-\frac{1}{2}, \frac{1}{2}\right)^{n} \text {. }
$$

In [1], we give the following two results about radix representations.

Theorem 3. Let $A$ be an $n \times n$ dilation matrix. If $\mu>2$, then $A$ yields a radix representation of $\mathbb{Z}^{n}$ with digit set $D=A(F) \cap \mathbb{Z}^{n}$.

Corollary 4. For every dilation matrix $A$, there exists a positive integer $\beta \geq 1$ such that $A^{\beta}$ yields a radix representation with digit set $D_{\beta}=A^{\beta}(F) \cap \mathbb{Z}^{n}$.

\section{RADIX REPRESENTATIONS AND TILINGS}

Radix representations are closely related to self-affine tilings of $\mathbb{R}^{n}$.

Definition 5. A measurable set $Q \subset \mathbb{R}^{n}$ gives a self-affine tiling of $\mathbb{R}^{n}$ under translation by $\mathbb{Z}^{n}$ if

(1) $\bigcup_{k \in \mathbb{Z}^{n}}(Q+k)=\mathbb{R}^{n}$, and the intersection $\left(Q+k_{1}\right) \cap\left(Q+k_{2}\right)$ has measure zero for any two distinct $k_{1}, k_{2} \in \mathbb{Z}^{n}$ (tiling); and

(2) there is a collection of $q=|\operatorname{det} A|$ vectors $k_{1}, \ldots, k_{q} \in \mathbb{Z}^{n}$ that are distinct coset representatives of $\mathbb{Z}^{n} / A\left(\mathbb{Z}^{n}\right)$ such that

$$
\left.A(Q) \simeq \bigcup_{i=1}^{q}\left(Q+k_{i}\right) \quad \text { (self-affine }\right)
$$

Set

$$
T=T(A, D):=\left\{\xi \in \mathbb{R}^{n}: \xi=\sum_{j=1}^{\infty} A^{-j} d_{j}\right\}
$$

with the digits $d_{j} \in D$ for some digit set $D$. One can easily check that $T$ is a selfaffine set. We would like to be able to think of the elements of $T$ as the fractional parts of vectors in $\mathbb{R}^{n}$ in the same way that the fractional parts of real numbers lie in $[0,1]$. This is an accurate interpretation if $T$ is congruent to $\mathbb{R}^{n} / \mathbb{Z}^{n}$. Thought of another way, we would like $T$ to tile $\mathbb{R}^{n}$ under translation by $\mathbb{Z}^{n}$.

Theorem 6. Let $A$ be a dilation matrix, and let $D$ be a digit set for $A$. Then $A$ yields a radix representation with digit set $D$ if and only if the set $T(A, D)$ tiles $\mathbb{R}^{n}$ under translation by $\mathbb{Z}^{n}$ and the origin $\mathbf{0}$ is in the interior of $T$. 
We split the proof of this theorem into a few lemmas.

Lemma 7. Let $A$ be a dilation matrix, and let $D$ be a digit set for $A$. If $A$ yields a radix representation with a digit set $D$, then the set $T(A, D)$ tiles $\mathbb{R}^{n}$ under translation by $\mathbb{Z}^{n}$.

Proof. Applying Proposition 5.19 from [12] the dilation matrix $A$, digit set $D$, and corresponding set $T=T(A, D)$ satisfy

(1) $T$ is a compact subset of $\mathbb{R}^{n}$;

(2) $A(T)=\bigcup_{d \in D}(T+d)$;

(3) $\bigcup_{x \in \mathbb{Z}^{n}}(T+x)=\mathbb{R}^{n}$; and

(4) $T$ contains an open set.

To show that $T$ tiles $\mathbb{R}^{n}$ under translation by $\mathbb{Z}^{n}$, we must show that

$$
m((T+x) \cap(T+y))=0 \text { for all } x \neq y, x, y \in \mathbb{Z}^{n}
$$

(where $m(\cdot)$ denotes Lebesgue measure). We extend an idea of Lagarias and Wang ([6], p. 31) to show that $m((T+x) \cap(T+y))=0$ for any distinct $x, y \in \mathbb{Z}^{n}$ for which there is a radix representation with radix $A$ and digit set $D$.

Note that for any subset $Q$ of $\mathbb{R}^{n}, m(A(Q))=q m(Q)$ (where $\left.q=|\operatorname{det} A|\right)$. In particular,

$$
\begin{aligned}
q m(T) & =m(A(T))=m\left(\bigcup_{d \in D}(T+d)\right)(\text { by property }(2) \text { of } T) \\
& \leq \sum_{d \in D} m(T+d)=\sum_{d \in D} m(T)=q m(T) .
\end{aligned}
$$

Additionally, property (2) implies that $A^{k+1}(T)=\bigcup_{d \in D}\left(A^{k}(T)+A^{k} d\right)$ for all $k \geq 0$. Then

$$
q^{k+1} m(T) \leq \sum_{d \in D} m\left(A^{k}(T)+A^{k} d\right)=\sum_{d \in D} q^{k} m(T)=q^{k+1} m(T) .
$$

Thus

$$
m\left(\left(A^{k}(T)+A^{k} d_{i}\right) \cap\left(A^{k}(T)+A^{k} d_{j}\right)\right)=0
$$

for all distinct $d_{i}, d_{j} \in D$.

Since $\mathbf{0} \in D, A^{k+1}(T) \supset A^{k}(T)$ for all $k \geq 0$. So

$$
\left(A^{k+1}(T)+A^{k+1} d\right) \supset\left(A^{k}(T)+A^{k+1} d\right)
$$

for all $d \in D$. Now consider $T+x$ for any $x \in \mathbb{Z}^{n}$. By hypothesis,

$$
x=A^{N} d_{N}+\sum_{j=0}^{N-1} A^{j} d_{j}
$$

for some $N \geq 0$, with $d_{N} \neq \mathbf{0} \in D$ and $d_{j} \in D$. Then

$$
\begin{aligned}
T+x & =\left\{y \in \mathbb{R}^{n}: y=A^{N} d_{N}+\sum_{j=0}^{N-1} A^{j} d_{j}+\sum_{j=-\infty}^{-1} A^{j} d_{j}, \text { all } d_{j} \in D\right\} \\
& \subset A^{N-1}(T)+A^{N} d_{N} \subset A^{N}(T)+A^{N} d_{N}
\end{aligned}
$$

and

$$
(T \cap(T+x)) \subseteq\left(A^{N}(T) \cap\left(A^{N}(T)+A^{N} d_{N}\right)\right)
$$




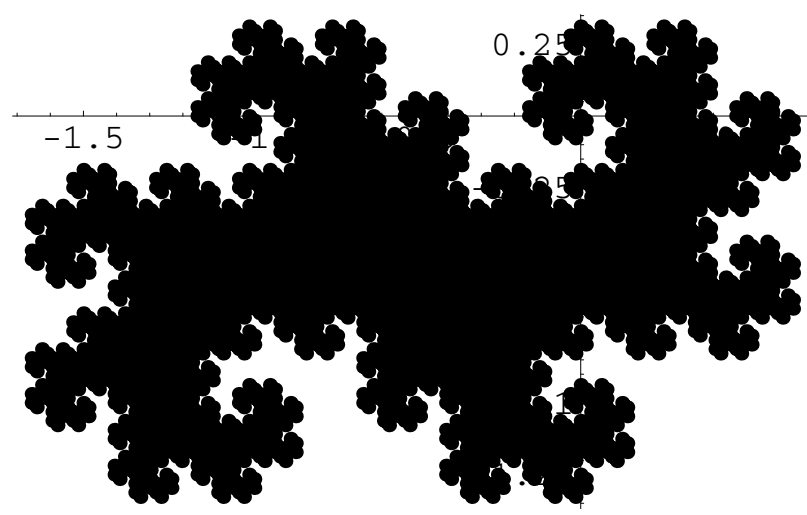

Figure 1. The tile $T$ for the twin dragon matrix.

Then $d_{N} \neq \mathbf{0} \in D$ implies that

$$
0=m\left(A^{N}(T) \cap\left(A^{N}(T)+A^{N} d_{N}\right)\right) \geq m(T \cap(T+x)) .
$$

If the set $T=T(A, D)$ tiles $\mathbb{R}^{n}$, it is not necessarily true that $A$ yields a radix representation. For example, in the case where $A=2$, we can find a radix representation for all nonnegative integers with the digit set $D=\{0,1\}$, or for all non-positive integers with the digit set $D=\{0,-1\}$, but we cannot represent all integers with a radix representation using any digit set [10]. Yet $T=[0,1]$ (with digit set $D=\{0,1\}$ ) does tile $\mathbb{R}$ under translation by $\mathbb{Z}$. Similarly, if $A$ is the twin dragon matrix [12],

$$
A=\left[\begin{array}{rr}
1 & 1 \\
-1 & 1
\end{array}\right]
$$

then $A$ is a dilation matrix. A digit set for $A$ is

$$
D=\left\{d_{0}=\left[\begin{array}{l}
0 \\
0
\end{array}\right], d_{1}=\left[\begin{array}{l}
1 \\
0
\end{array}\right]\right\},
$$

and the set $T$ generated by the twin dragon matrix with this digit set tiles $\mathbb{R}^{2}$ under translation by $\mathbb{Z}^{n}\left[12\right.$, yet $A$ does not yield a radix representation of $\mathbb{Z}^{n}$ (for example, the vector

$$
\left[\begin{array}{r}
0 \\
-1
\end{array}\right]
$$

does not have a radix representation) 1 .

In both of the examples, the origin $\mathbf{0}$ is on the boundary of the tile $T$, so that $\bigcup_{k=0}^{\infty} A^{k}(T) \subsetneq \mathbb{R}^{n}$. We claim in Theorem 6 that this must be the case for all dilation matrices $A$ which give tiles $T$ but do not yield radix representations. We first introduce a technical lemma.

Lemma 8. Let $A$ be a dilation matrix, and let $D$ be a digit set for $A$. If $\mathbf{0} \notin T^{\circ}$, then there exists an increasing subsequence $\left\{\ell_{j}\right\}_{j \geq 1}$ of the positive integers and a sequence of vectors $\left\{\zeta_{j}: \zeta_{j} \in A^{-\ell_{j}}\left(\mathbb{Z}^{n}\right)\right\}_{j \geq 1}$ converging to $\mathbf{0}$ such that $\zeta_{j} \notin T$ for all $j \geq 1$. 
Proof. In the proof of Lemma 7 , we note that the set $T$ is compact. Thus for any $\omega \in \mathbb{R}^{n}$ such that $\omega \notin T, d(\omega, T)>0$, and there exists an open ball centered at $\omega$, $B_{\omega}$, such that $\overline{B_{\omega}} \cap T=\emptyset$.

Let $\left\{y_{j}\right\}_{j \geq 1}$ be a sequence of vectors in $\mathbb{R}^{n}$ converging to $\mathbf{0}$ with $y_{j} \notin T$ for all $j \geq 1$. Set $\epsilon_{j}=\left\|y_{j}\right\|_{l^{2}}$, and note that $\epsilon_{j} \geq d\left(y_{j}, T\right)>0$, with $\lim _{j \rightarrow \infty} \epsilon_{j}=0$.

Set $r_{1}=\frac{d\left(y_{1}, T\right)}{2}$ and

$$
r_{j}=\min \left\{\frac{r_{j-1}}{2}, \frac{d\left(y_{j}, T\right)}{2}\right\}
$$

for $j \geq 2$. Then $\left\{r_{j}\right\}_{j \geq 1}$ is a decreasing sequence of positive numbers, $\lim _{j \rightarrow \infty} r_{j}=$ 0 , and the open balls $B_{j}$ of radius $r_{j}$ centered at the vectors $y_{j}$ satisfy $\overline{B_{j}} \cap T=\emptyset$ for all $j \geq 1$. By construction, if $y_{j}^{*}$ is any point in the ball $B_{j}$ for each $j \geq 1$, then $\lim _{j \rightarrow \infty} y_{j}^{*}=\mathbf{0}$ and $y_{j}^{*} \notin T$ for all $j \geq 1$.

In particular, there exists an increasing subsequence $\left\{\ell_{j}\right\}_{j \geq 1}$ of the positive integers such that $A^{-\ell_{j}}$ is a fine enough lattice to ensure that $A^{-\ell_{j}}\left(\mathbb{Z}^{n}\right) \cap B_{j} \neq \emptyset$ for each $j \geq 1$. We may choose some $\zeta_{j} \in A^{-\ell_{j}}\left(\mathbb{Z}^{n}\right) \cap B_{j}$ for each $j \geq 1$. By construction, $\zeta_{j} \notin T$ for each $j \geq 1$, and the sequence $\left\{\zeta_{j}\right\}_{j \geq 1}$ converges to $\mathbf{0}$.

Lemma 9. Let $A$ be a dilation matrix, and let $D$ be a digit set for $A$. If $A$ yields a radix representation with digit set $D$, then $\mathbf{0}$ is in the interior of the set $T$ generated by $A$ and $D$.

Proof. We prove the desired result by contradiction. Assume that $\mathbf{0} \notin T^{\circ}$. By Lemma 8 , let $\left\{\zeta_{j}\right\}_{j \geq 1}$ be a sequence of vectors in $\mathbb{R}^{n}$ converging to $\mathbf{0}$ such that $\zeta_{j} \in A^{-\ell_{j}}\left(\mathbb{Z}^{n}\right)$ (for some increasing subsequence $\left\{\ell_{j}\right\}_{j \geq 1}$ of the positive integers) but $\zeta_{j} \notin T$ for all $j \geq 1$. We may write $\zeta_{j}=A^{-\ell_{j}} x_{j}$ for some $x_{j} \in \mathbb{Z}^{n}$ for each $j$. Since $A$ yields a radix representation, there exists an integer $N_{j}$ for each $x_{j}$ and $\operatorname{digits} d_{0}^{(j)}, \ldots, d_{N_{j}}^{(j)} \in D$ such that

$$
x_{j}=\sum_{i=0}^{N_{j}} A^{i} d_{i}^{(j)} .
$$

Thus

$$
\zeta_{j}=\sum_{i=0}^{N_{j}} A^{i-\ell_{j}} d_{i}^{(j)}=k_{j}+\xi_{j}
$$

with $k_{j} \in \mathbb{Z}^{n}$ and $\xi_{j} \in T$.

Since $T$ is compact, $\left\|\xi_{j}\right\|_{l^{2}}$ is bounded above by $b$ for some $b>0$. Then, since $\zeta_{j}$ converges to $\mathbf{0},\left\|k_{j}\right\|_{l^{2}}$ is also bounded above for sufficiently large $j$. We use the rough estimate that there exists an integer $M \geq 1$ such that for all $j \geq M$, $\left\|k_{j}\right\|_{l^{2}} \leq 2 b$. Thus for $j \geq M$, the integer vectors $k_{j}$ all belong to a finite subset of $\mathbb{Z}^{n}$. This implies that there exists an integer $N \geq 0$ such that $N_{j} \leq N$ for all $j \geq M$. For $j>\max \{M, N\}, N-\ell_{j} \leq N-j<0$, and thus $\zeta_{j} \in T$ for all sufficiently large $j$. This contradicts the choice of $\zeta_{j}$, thus our assumption that $\mathbf{0} \notin T^{\circ}$ must be false.

We have shown that if a dilation matrix $A$ yields a radix representation with digit set $D$, then the set $T=T(A, D)$ tiles $\mathbb{R}^{n}$ under translation by $\mathbb{Z}^{n}$, and $\mathbf{0} \in T^{\circ}$. Next we prove the converse. 
Lemma 10. Let $A$ be a dilation matrix, and let $D$ be a digit set for $A$. If the set $T=T(A, D)$ tiles $\mathbb{R}^{n}$ under translation by $\mathbb{Z}^{n}$ and if $\mathbf{0} \in T^{\circ}$, then $A$ yields a radix representation with digit set $D$.

Proof. Following the notation of [6], set

$$
D_{A, k}:=\left\{x \in \mathbb{Z}^{n}: x=\sum_{j=0}^{k-1} A^{j} d_{j}, d_{j} \in D\right\},
$$

the set of vectors that can be expressed with a radix representation of length less than or equal to $k$. Note that $D_{A, 1}=D$. By construction, $A(T)=\bigcup_{d \in D}(T+d)$. Thus

$$
A^{k}(T)=\bigcup_{x \in D_{A, k}}(T+x)
$$

By the tiling hypothesis, $\left(T^{\circ}+x\right) \cap\left(T^{\circ}+y\right)=\emptyset$ for all distinct $x, y \in \mathbb{Z}^{n}$. Thus $\left(T^{\circ}+y\right) \cap A^{k}\left(T^{\circ}\right)=\emptyset$ for all $y \in \mathbb{Z}^{n}$ with $y \notin D_{A, k}$, and

$$
D_{A, k} \supseteq\left(A^{k}\left(T^{\circ}\right) \cap \mathbb{Z}^{n}\right) .
$$

Let $B$ be an open ball centered at the origin such that $B \subseteq T^{\circ}$. Then

$$
D_{A, k} \supseteq\left(A^{k}(B) \cap \mathbb{Z}^{n}\right) .
$$

The sets $A^{k+1}(B)$ are expanding, with $\bigcup_{k \geq 0} A^{k+1}(B)=\mathbb{R}^{n}$. Thus

$$
\bigcup_{k \geq 0} D_{A, k} \supseteq \bigcup_{k \geq 0}\left(A^{k}(B) \cap \mathbb{Z}^{n}\right)=\mathbb{Z}^{n} .
$$

The opposite containment is true as well, since $D_{A, k} \subset \mathbb{Z}^{n}$ for each $k$. Thus $\bigcup_{k \geq 0} D_{A, k}=\mathbb{Z}^{n}$.

We have now completed the proof of Theorem 6 .

Recall that $\mu$ is the smallest singular value of the dilation matrix $A$ and that $F$ is our canonical fundamental domain of $\mathbb{Z}^{n}, F=\left[-\frac{1}{2}, \frac{1}{2}\right)^{n}$. Combining Theorems 3 and Lemma 7, we also have the following corollary.

Corollary 11. Let $A$ be a dilation matrix, and let $D=A(F) \cap \mathbb{Z}^{n}$. If $\mu>2$, then the set

$$
T=\left\{x \in \mathbb{R}^{n}: x=\sum_{j=-\infty}^{-1} A^{j} d_{j}, d_{j} \in D\right\}
$$

tiles $\mathbb{R}^{n}$ under translation by $\mathbb{Z}^{n}$.

\section{HAAR-Like WAVELETS}

Self-affine tiles allow us to construct multivariable wavelet sets associated with multiresolution analyses. Here we review some basic definitions from wavelet theory.

Definition 12. A multiresolution analysis (MRA) associated with a dilation matrix $A$ is a nested sequences of subspaces $\cdots \subset V_{-1} \subset V_{0} \subset V_{1} \subset \cdots$ of $L^{2}\left(\mathbb{R}^{n}\right)$ satisfying [12]:

(1) $\overline{\bigcup_{j \in \mathbb{Z}} V_{j}}=L^{2}\left(\mathbb{R}^{n}\right)$;

(2) $\bigcap_{j \in \mathbb{Z}} V_{j}=\{0\}$;

(3) $f(x) \in V_{j}$ if and only if $f(A x) \in v_{j+1}$ for all $j \in \mathbb{Z}$;

(4) $f(x) \in V_{0}$ if and only if $f(x-k) \in V_{0}$ for all $k \in \mathbb{Z}^{n}$; and 
(5) there exists a function $\phi(x) \in V_{0}$, called a scaling function, such that

$$
\left\{\phi(x-k): k \in \mathbb{Z}^{n}\right\}
$$

is a complete orthonormal basis for $V_{0}$.

The existence of multiresolution analyses in dimension $n>1$ has been studied by a number of authors. In [3], Gröchenig and Madych showed that if $\phi=$ $(m(Q))^{1 / 2} \chi_{Q}$ is a scaling function for a multiresolution analysis, then $Q$ must be an affine image of a self-affine tiling of $\mathbb{R}^{n}$ under translation by $\mathbb{Z}^{n}$. They showed also that if $T$ is a set of the form

$$
T=\left\{x \in \mathbb{R}^{n}: x=\sum_{j=-\infty}^{-1} A^{j} d_{j}, d_{j} \in D\right\}
$$

with $A$ a dilation matrix and $D$ a digit set for $A$, then $\phi=\chi_{T}$ is the scaling function for a multiresolution analysis (note that $|T|=1$ ). Lagarias and Wang noted that all self-affine tiles $T$ which tile $\mathbb{R}^{n}$ under translation by $\mathbb{Z}^{n}$ must be of this form [8] A scaling function that is the characteristic function of some measurable set is called a Haar-like scaling function (after the Haar scaling function, which is $\chi_{[0,1]}$ ).

Lagarias and Wang studied necessary conditions for sets of the form $T(A, D)$ to tile $\mathbb{R}^{n}$ under translation by $\mathbb{Z}^{n}$ in a series of papers $(6,6], 8,[9)$. Much of their work in these papers concerned the question of when a set $T(A, D)$ tiles $\mathbb{R}^{n}$ under translation by a sublattice of $\mathbb{Z}^{n}$. In [6] and [7, they showed that if $D$ is a complete set of coset representatives of $\mathbb{Z}^{n} / A\left(\mathbb{Z}^{n}\right)$, then $T(A, D)$ is a self-affine tile of $\mathbb{R}^{n}$ under translation by some sublattice of $\mathbb{Z}^{n}$. They also studied some properties of the tiling set $T(A, D)$. He and Lau [4] studied sets $T(A, D)$ which tile $\mathbb{R}^{n}$ under translation by a sublattice of $\mathbb{Z}^{n}$ as well; in particular, they looked at possible digit sets $D$.

In order for $\chi_{T}$ to be a scaling function for a multiresolution analysis, however, we need $T$ to tile $\mathbb{R}^{n}$ under translation by all of $\mathbb{Z}^{n}$. Lagarias and Wang gave some necessary conditions for a dilation matrix $A$ to yield a Haar-like scaling function in 8 and 9. They showed that all dilation matrices in dimensions $n=2$ and 3 yield Haar-like scaling functions. Our results below give a sufficient condition for a dilation matrix $A$ to yield a Haar-like scaling function, in any dimension. Note that for dilation matrices that yield a Haar-like scaling function, Strichartz has shown that multiresolution analyses and associated wavelet bases with arbitrary regularity can be constructed 11.

The results of the previous section imply the following two theorems.

Theorem 13. Let $A$ be a dilation matrix, and let $D$ be a digit set for $A$ such that $A$ yields a radix representation for $\mathbb{Z}^{n}$ with digit set $D$. Let $T$ be the set depending on $A$ and $D$ defined above. Then $\phi=\chi_{T}$ is the scaling function for a multiresolution analysis. In particular, if $A$ satisfies $\mu>2$ and if $D$ is the set $D=A(F) \cap \mathbb{Z}^{n}$ with $F=\left[-\frac{1}{2}, \frac{1}{2}\right)^{n}$, then $\phi=\chi_{T}$ is the scaling function for a multiresolution analysis.

Theorem 14. Let $A$ be a dilation matrix. Then there exists a positive integer $\beta \geq 1$ such that for all integers $k \geq \beta$ there exists a multiresolution analysis associated with the dilation matrix $A^{k}$.

Thus Haar-like scaling functions and associated MRAs exist for a large class of dilation matrices. 


\section{REFERENCES}

[1] E. Curry, Radix and Pseudodigit Representations in $\mathbb{Z}^{n}$, preprint.

[2] Julie Belock, Vladimir Dobric, Random Variable Dilation Equation and Multidimensional Prescale Functions, Trans. Amer. Math. Soc. 353 (2001), 4779-4800. MR1852082 (2002g:60003)

[3] K. Gröchenig and W.R. Madych, Multiresolution Analysis, Haar Bases, and Self-Similar Tilings of $\mathbb{R}^{n}$, IEEE Trans. Info. Thy. 38 (1992), 556-568. MR1162214 (93i:42001)

[4] Xing-Gang He and Ka-Sing Lau, Characterization of tile digit sets with prime determinants, Appl. Comput. Harmon. Anal. 16 (2004), 159-173. MR2054276 (2005c:52019)

[5] E.-C. Jeong, A Number System in $\mathbb{R}^{n}$, J. Korean Math. Soc. 41 (2004), 945-955. MR2095546 (2005f:11013)

[6] Jeffrey C. Lagarias and Yang Wang, Self-Affine Tiles in $\mathbf{R}^{n}$, Adv. in Math. 121 (1996), 21-49. MR1399601 (97d:52034)

[7] Jeffrey C. Lagarias and Yang Wang, Integral Self-Affine Tiles in $\mathbb{R}^{n}$ Part II: Lattice Tilings, J. Fourier Anal. and Appl. 3 (1997), 83-102. MR.1428817 (98b:52026)

[8] Jeffrey C. Lagarias and Yang Wang, Haar Bases for $L^{2}\left(\mathbb{R}^{n}\right)$ and Algebraic Number Theory, J. Number Thy. 57 (1996), 181-197. MR1378581 (97a:42027)

[9] Jeffrey C. Lagarias and Yang Wang, Corrigendum/Addendum: Haar Bases for $L^{2}\left(\mathbb{R}^{n}\right)$ and Algebraic Number Theory, J. Number Thy. 76 (1999), 330-336. MR1684691 (2000g:42042)

[10] David W. Matula, Basic Digit Sets for Radix Representation, J. Assoc. Comp. Machinery 29 (1982), 1131-1143. MR0674260 (83k:68017)

[11] R. Strichartz, Wavelets and self-affine tilings, Constr. Approx. 9 (1993), 327-346. MR.1215776 (94f:42039)

[12] P. Wojtaszczyk, "A Mathematical Introduction to Wavelets." Cambridge University Press, Cambridge, United Kingdom, 1997. MR1436437 (98j:42025)

Department of Mathematics and Statistics, Dalhousie University, Halifax, Nova ScoTIA, CANADA B3H 3J5

E-mail address: ecurry@mathstat.dal.ca 